\title{
Application of 3D printed osteotomy guide plate-assisted total knee arthroplasty in treatment of valgus knee deformity
}

\author{
Zhimin Shen ${ }^{\dagger}$, Hong Wang ${ }^{\dagger}$, Yiqiang Duan, Jian Wang ${ }^{*}$ and Fengyan Wang
}

\begin{abstract}
Introduction: To analyze the application of 3D printed osteotomy guide plate-assisted total knee arthroplasty (TKA) for valgus knee deformity.

Methods: The clinical data of 20 patients with valgus knee deformity admitted to our hospital from April 2012 to April 2017 were collected and analyzed. According to the treatment method, these patients were divided into two groups: 3D printed osteotomy guide plate-assisted TKA (combined treatment group, $n=10$ ) and TKA (treatment group, $n=10$ ). The operation time, intraoperative bleeding volume, postoperative mean femorotibial angle (MFTA), and Knee Society Score (KSS) of the two groups were statistically analyzed.
\end{abstract}

Results: Compared with the treatment group, the operation time was significantly shorter $(P<0.05)$, the intraoperative blood loss and postoperative MFTA were significantly decreased $(P<0.05)$, and the clinical and functional scores were significantly increased $(P<0.05)$ in the combined treatment group.

Conclusion: 3D printed osteotomy guide plate-assisted TKA for valgus knee deformity is more effective than TKA alone.

Keywords: 3D printed osteotomy guide plate-assisted total knee arthroplasty, Valgus knee deformity, Clinical operation

\section{Introduction}

Total knee arthroplasty (TKA) is the most effective treatment of knee pain and dysfunction, which can reduce the pain of patients, restore the lower limb line, and reconstruct joint function [1]. It is also the gold standard procedure with excellent results for the treatment of advanced knee arthritis [2]. However, it is well known that valgus deformity with tibial plateau bone defect and dysplasia of the femoral condyle can lead to increased difficulty in TKA [3].

Valgus knee deformity is defined as a valgus angle equal to or greater than $10^{\circ}$ and is observed in nearly $10 \%$ of patients undergoing TKA [4]. Excessive preoperative malalignment predisposes to a greater risk of failure [4]. The long-term results in valgus deformed knee were relatively inferior to varus deformation. One

\footnotetext{
* Correspondence: jian_w19@outlook.com

Zhimin Shen and Hong Wang contributed equally to this study Department of Orthopedics, The Affiliated Hospital of Guizhou Medical University, No. 28, Guiyijie Road, Guiyang City 550004, Guizhou Province, China
}

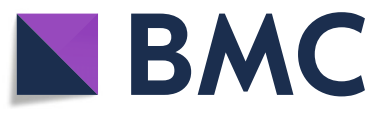

(c) The Author(s). 2019 Open Access This article is distributed under the terms of the Creative Commons Attribution 4.0 International License (http://creativecommons.org/licenses/by/4.0/), which permits unrestricted use, distribution, and reproduction in any medium, provided you give appropriate credit to the original author(s) and the source, provide a link to the Creative Commons license, and indicate if changes were made. The Creative Commons Public Domain Dedication waiver (http://creativecommons.org/publicdomain/zero/1.0/) applies to the data made available in this article, unless otherwise stated. soft tissue balance during surgery [2].

$3 \mathrm{D}$ printing allows a surgeon to better visualize the anatomy in full 3D and digitally plan the osteotomy preoperatively based on CT images, resulting in improved accuracy of preoperative planning and surgical precision and decreased postoperative complication ratio [5-7]. It improves the accuracy of surgical resection by avoiding large segmental bone defects, improves the stability of knee joint after reconstruction, and improves the mechanical strength and stability of the prosthesis [8]. This technique is very precise and promising especially in patients requiring limb corrections in multiple planes [5]. At present, 3D printing technology has been used in the production of orthopedic experimental model, surgical auxiliary material printing, and printing of implant and joint surgery [9].

In this study, the operation time, intraoperative bleeding volume, mean femorotibial angle (MFTA), and Knee Society Score (KSS) between the application of 3D printed 
Table 1 Comparison of general data between the two groups of patients

\begin{tabular}{|c|c|c|c|c|c|}
\hline Item & Classification & Combined treatment group $(n=10)$ & Treatment group $(n=10)$ & $t / x^{2}$ & $P$ \\
\hline \multirow[t]{2}{*}{ Gender } & Male & $2(20.0)$ & $1(10.0)$ & 2.71 & $>0.05$ \\
\hline & Female & $8(80.0)$ & $9(90.0)$ & & \\
\hline Age (year) & & $60.3 \pm 10.1(59-71)$ & $61.4 \pm 10.2(60-71)$ & 1.886 & $>0.05$ \\
\hline \multirow[t]{2}{*}{ Primary diseases } & Osteoarthritis & $6(60.0)$ & $5(50.0)$ & 1.32 & $>0.05$ \\
\hline & Rheumatoid arthritis & $4(40.0)$ & $5(50.0)$ & & \\
\hline \multirow[t]{3}{*}{ Keblish grading } & Mild & $5(50.0)$ & $4(40.0)$ & 2.77 & $>0.05$ \\
\hline & Moderate & $4(40.0)$ & $4(40.0)$ & & \\
\hline & Severe & $1(10.0)$ & $2(20.0)$ & & \\
\hline
\end{tabular}

Data were presented as mean $\pm S D, n$ (percentage) or range. $n$ number of cases

osteotomy guide plate-assisted TKA and artificial TKA for valgus knee deformity were compared.

\section{Materials and methods}

\section{General information}

The clinical data of 20 patients with valgus knee deformity admitted to our hospital from April 2012 to April 2017 were collected and analyzed. Inclusive criteria are as follows: all patients who were operated for the first time. Routine axial X-ray examination of the patella was performed before operation. Exclusive criteria are as follows: patients with surgical contraindications. According to the treatment method, these patients were divided into two groups: 3D printed osteotomy guide plate-assisted TKA (combined treatment group, $n=10$ ) and TKA (treatment group, $n=10$ ). There were 2 males and 8 females in the combined treatment group, aged 59-71 years, with an average of $60.3 \pm 10.1$ years old. According to their primary diseases, 6 patients had osteoarthritis and 4 patients had rheumatoid arthritis. According to the Keblish classification [10], 5 cases were mild, 4 cases were moderate, and 1 case was severe. There were 1 male and 9 females in the TKA treatment group, aged $60-71$ years, mean
$61.4 \pm 10.2$ years old. According to their primary diseases, 5 patients had osteoarthritis and 5 patients had rheumatoid arthritis. In terms of the Keblish classification, 4 cases were mild, 4 cases were moderate, and 2 cases were severe. The general data were comparable between the two groups $(P>0.05)$ (Table 1$)$. This study was approved by the Ethics Committee of the authors' hospital, and informed consent was taken prior to enrollment.

\section{Methods \\ Main instruments}

The main instruments used were as shown in Table 2.

\section{Treatment group}

Patients in the treatment group received artificial TKA. The specific operation was as follows: Under general anesthesia, the patients were placed in a supine position, and an airbag tourniquet was used to pressurize the blood. The medial approach of the iliac crest was applied. First, routine femoral condyle and tibia plateau osteotomies were performed. Then, the posterior lateral joint capsule and lateral iliotibial band were completely loosened by using a pie-crusting technique. The

Table 2 Main instruments

\begin{tabular}{|c|c|c|}
\hline Type of instrument & Conditions of use, materials, and characteristics & Source \\
\hline Mimics 10.0 & $\begin{array}{l}\text { For processing images and creating 3D models. Can be used to import DICOM } \\
\text { or raw image data, export 3D models for analysis, design or 3D printing, virtually } \\
\text { plan a surgical procedure, create 3D models, and perform anatomical analysis. } \\
\text { Uses } 2 \mathrm{D} \text { cross-sectional images such as from } \mathrm{CT} \text { to construct 3D models, which } \\
\text { can then be directly linked to RP, CAD, and surgical simulation. }\end{array}$ & Materialise, Leuven, Belgium \\
\hline Creator Pro 3D printer & $\begin{array}{l}\text { For creating 3D models. Equipped with versatile dual extruder, solid steel frame } \\
\text { construction, and has a stable vertical movement. }\end{array}$ & $\begin{array}{l}\text { FlashForge, Zhejiang, People's } \\
\text { Republic of China }\end{array}$ \\
\hline $\begin{array}{l}\text { 64-row 128-slice volume } \\
\text { CT (VCT) }\end{array}$ & $\begin{array}{l}\text { For image acquisition. The scanning range includes the target knee joint. } \\
\text { Scanning parameters: collimator } 128 \times 0.5 \mathrm{~mm} \text {; tube voltage } 120 \mathrm{kV} \text {, the tube } \\
\text { current was automatically adjusted according to the patient's body thickness; } \\
\text { rotation time } 0.5 \mathrm{~s} / \mathrm{r} \text {; layer thickness } 3 \mathrm{~mm} \text {, layer spacing } 3 \mathrm{~mm} \text {, reconstruction } \\
\text { with soft tissue algorithm and bone algorithm, FOV }=512 \times 512 \text {. }\end{array}$ & $\begin{array}{l}\text { SOMATOM Definition AS, } \\
\text { Siemens AG, Erlangen, Germany }\end{array}$ \\
\hline $\begin{array}{l}\text { Knee replacement prosthesis } \\
\text { and surgical instruments }\end{array}$ & The knee replacement prosthesis included the femoral and tibial components. & DePuy Synthes, Raynham, MA, USA \\
\hline CAD software (2012 version) & For modeling, measuring the digital model, and simulating surgery. & Autodesk Inc., San Rafael, CA, USA \\
\hline
\end{tabular}




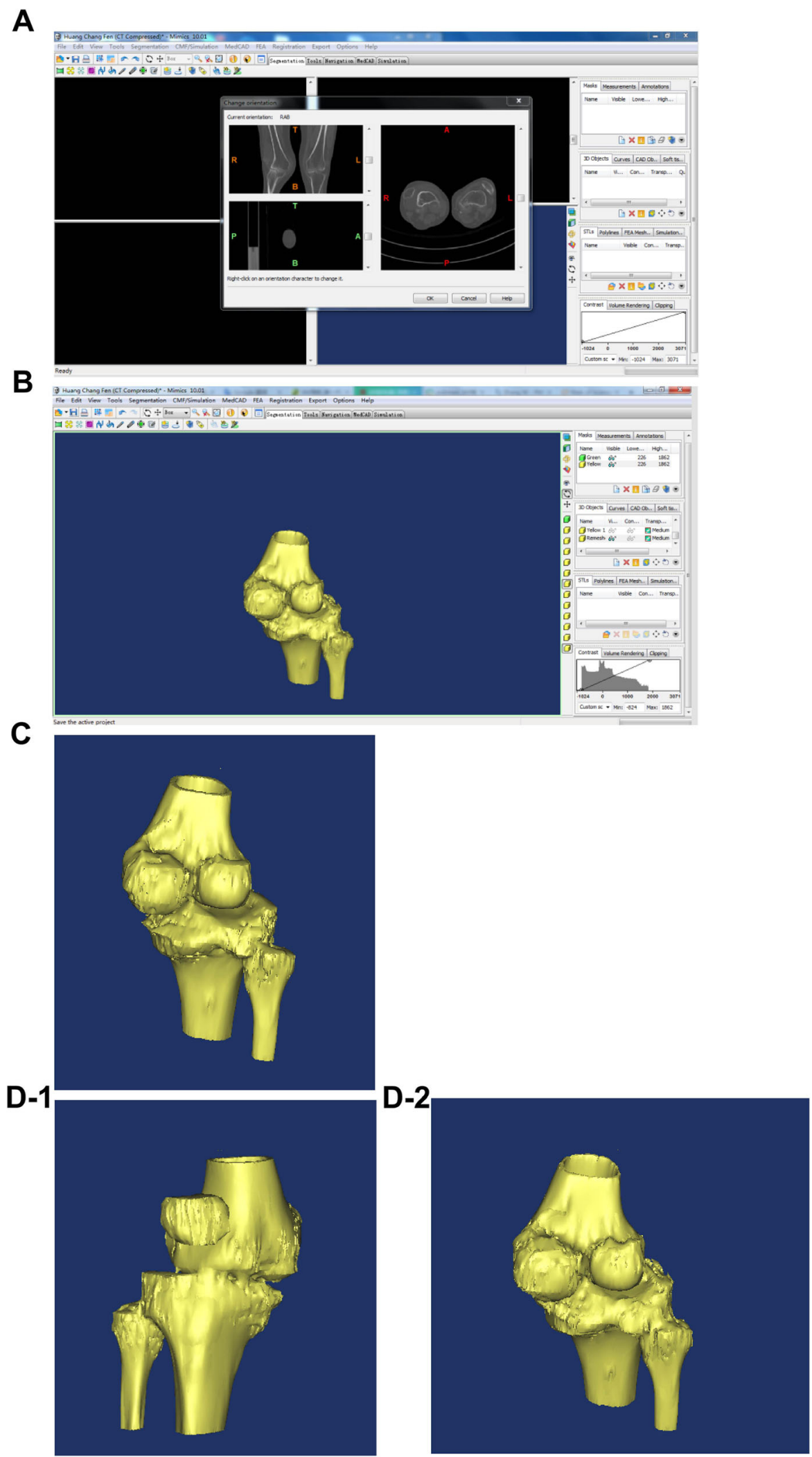

Fig. 1 A Input data into Mimics software imaging. B Formation of 3D printed knee joint image in Mimics software. C Demonstration of 3D knee joint generated via Mimics software. Knee joint image generated D-1 anterior-posterior view and D-2 posterior view 


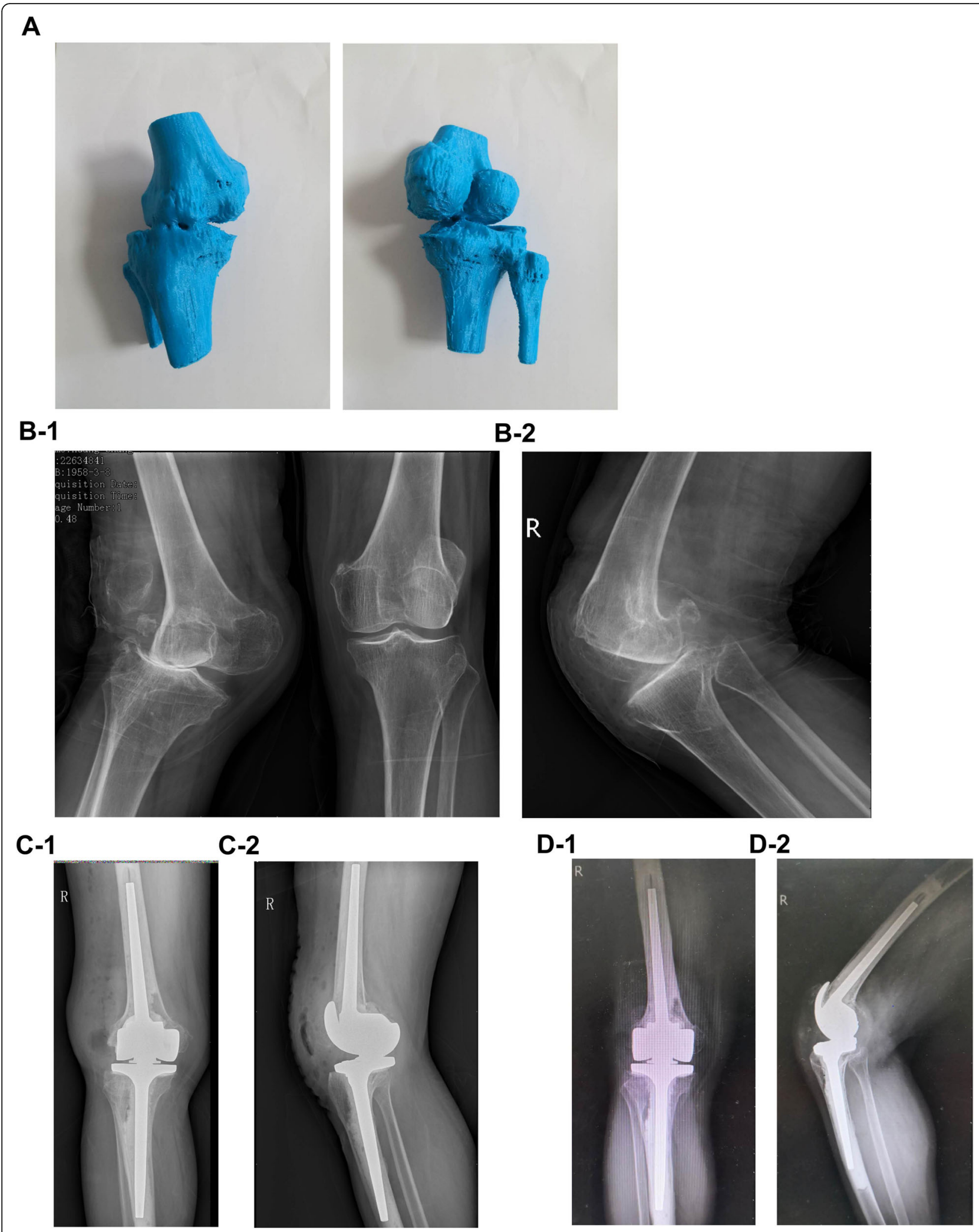

Fig. 2 A 3D printing model. Preoperative anterior-posterior view (B-1) and lateral view (B-2). Postoperative anterior-posterior view (C-1) and lateral view (C-2). 1-year postoperative anterior-posterior view (D-1) and lateral view (D-2) 
standard was set to the outer compartment tension, and the rectangular spacer with appropriate thickness was placed in the knee joint extension gap. If the knee joint was still unstable under valgus stress after the rectangular straightening gap and the accurate lower limb force line were obtained, the starting point was set in the medial collateral ligament, and the osteotomy was upwardly slid, thereby tightening the medial collateral ligament.

\section{Combined treatment group}

Patients in the combined treatment group received 3D printed osteotomy guide plate-assisted TKA. The procedure for artificial TKA was the same as above. The specific operation of the 3D printed osteotomy guide plate was as follows: CT image data from the patients of the femoral head to the ankle joint were collected before surgery to create the $3 \mathrm{D}$ printing model. In this process, the AutoCAD software was used to measure the digital model and simulate the surgery, a personalized osteotomy guide plate was then designed, and the patient's knee anatomical model and osteotomy guide plate digital file entity were printed. Through the internal/lateral approach of the iliac crest, the knee joint was exposed, the joint effusion was absorbed, the osteophytes such as synovial tissue and meniscus were removed, and the lateral soft tissue was moderately loosed. The patient's knee joint and 3D printed knee joint model were matched intraoperatively to eliminate loss of model accuracy caused by inaccurate image data or printing distortion. After determining the anatomy, the 3D femoral osteotomy guide plate was installed, ensuring the positioning module was attached to the anatomy of the knee joint. The femoral condyle was then osteotomied between the anterior and posterior oblique angles. Proximal tibia osteotomy was performed after the 3D tibial osteotomy guide plate was installed and was matched to the anatomical structure of the proximal tibia. The patient's soft tissue balance was re-examined and moderately adjusted and was then pulse flushed. After the patient had installed the prosthesis and spacer, the force line was inspected to ensure that there was a good force line. Then, drainage was placed and the wound was sutured. Figure 1 shows some steps involved in 3D printed osteotomy guide plate-assisted TKA. Figure 2 shows the $3 \mathrm{D}$ printing model and pre- and postoperative radiographs of a patient with valgus knee deformity.

\section{Observation index}

The operation time, intraoperative blood loss, and postoperative MFTA of the two groups were observed and measured. At the same time, the KSS was used to assess the clinical profile and function of the knee joint in the two groups before and after treatment. With the increase of score, the clinical profile and function of the knee joints were gradually improved [11].

\section{Statistical analysis}

The data were analyzed by IBM SPSS statistical software (version 20.0) (IBM Corp., Armonk, NY, USA) and expressed as mean \pm standard deviation $(\overline{\mathrm{x}} \pm \mathrm{s})$. All values were analyzed using analysis of variance (ANOVA) and Newman-Keuls-Student's $t$ test. $P<0.05$ was considered as statistically significant.

\section{Results}

Comparison of general data between the two groups

There was no significant difference in the general data between the two groups $(P>0.05$, Table 1$)$.

\section{Comparison of operation time, intraoperative blood loss, and postoperative MFTA between the two groups}

The operation time, intraoperative blood loss, and postoperative MFTA were significantly lower in the combination group than the treatment group $(P<0.05$, Table 3$)$.

\section{Comparison of KSS in the two groups before and after treatment}

The clinical and functional scores of KSS in both groups after treatment were significantly higher than those before treatment $(P<0.05)$. There was no significant difference in the KSS clinical and functional scores between the two groups before treatment $(P>0.05)$. The clinical and functional scores of the patients after treatment in the combined treatment group were significantly higher than those in the treatment group $(P<0.05$, Table 4$)$.

Table 3 Comparison of operation time, intraoperative blood loss, and postoperative MFTA between the two groups

\begin{tabular}{lllll}
\hline Group & $n$ & Operation time $(\mathrm{min})$ & Intraoperative blood loss $(\mathrm{ml})$ & Postoperative MFTA $\left(^{\circ}\right)$ \\
\hline Combined treatment group & 10 & $81.0 \pm 10.0^{*}$ & $246.4 \pm 43.3^{*}$ & $4.1 \pm 0.1^{*}$ \\
Treatment group & 10 & $87.0 \pm 10.2$ & $293.0 \pm 40.1$ & $5.6 \pm 0.7$ \\
$t$ & & 3.182 & 4.303 & 2.776 \\
$P$ & & $<0.05$ & $<0.05$ & $<0.05$ \\
\hline
\end{tabular}

Data were presented as mean \pm SD. $n$ number of cases

${ }^{*} P<0.05$, combined treatment group vs treatment group 
Table 4 Comparison of KSS scores in the two groups before or after treatment

\begin{tabular}{lllll}
\hline Group & $n$ & Time & Clinical score & Functional score \\
\hline Combined treatment group & 10 & Pretreatment & $23.2 \pm 3.1$ & $29.0 \pm 4.4$ \\
& & Posttreatment & $91.5 \pm 10.1^{\#^{*}}$ & $90.3 \pm 10.8^{\#^{*}}$ \\
Treatment group & 10 & Pretreatment & $23.0 \pm 5.1$ & $27.3 \pm 4.1$ \\
& & Posttreatment & $77.3 \pm 10.3^{\#}$ & $77.0 \pm 10.6^{\#}$ \\
\hline
\end{tabular}

Data were presented as mean \pm SD. $n$ number of cases

${ }^{\#} P<0.05$, posttreatment vs pretreatment

${ }^{*} P<0.05$, combined treatment group vs treatment group

\section{Discussion}

The desired thickness and angle of osteotomy which is determined by preoperative X-ray, intraoperative extramedullary positioning devices, and clinician's experience in routine TKA is easy to produce certain deviations, resulting in surgical failure [12]. Studies have shown that the accuracy of the osteotomy angle determined by conventional surgical instruments is only $75 \%$ compared with actual anatomy [13-17]. The coincidence rate will have a larger gap if the patient has severe cartilage loss and knee deformity. The 3D printed osteotomy guide plate takes full advantage of the two core technologies of rapid prototyping (RP) and computer-aided design (CAD). The CAD software can build a 3D model based on the patient's CT data. Meanwhile, it can measure, cut, and match anatomical models of patients [18]. In addition, CAD software can accurately measure anatomical structures before TKA and accurately reconstruct lower limb force lines [19]. It can be applied to precise digital orthopedics operation of bone and joint deformity [20], while increased precision are good indicators of how 3D guides reduce potential human error [21]. Furthermore, the 3D printed osteotomy guide plate can effectively assist in TKA operation [22].

To develop a 3D model based on the patient's CT data, first, the 64-row 128-slice VCT was used for scanning to obtain the thin-slice CT data. The relevant parameters were set according to the actual situation of each patient, and the obtained data were saved in Digital Imaging and Communications in Medicine (DICOM) format and reserved for use. Second, data transmission was conducted. The surgical transmission mode was selected to transmit the acquired data to Mimics 10.0 software. The "Import Image" command, import image and data, and lossless compression mode were selected, and according to the left and right orientation of the image and sagittal image, the anteriorposterior position was determined to complete the data import. Third, 2D image production was performed. In this study, bone window and soft tissue window scanning was used for CT data, and 3D reconstruction was performed. Combined with 2D images of the transverse plane, sagittal plane, and coronal plane, an appropriate threshold range was selected to separate the relevant tissues of the obtained images and generate the 2D bone tissue contour, which was the original mask. Fourth is the separation and editing of mask. On the basis of the original mask, the function of " $2 \mathrm{D}$ regional growth" was used to select the bone which connects with the main body, and combined with the transverse, sagittal, and coronal planes, the corresponding knee joint was selected and the 3D model of the knee joint mask was calculated. Fifth is the reconstructing of high simulation 3D model. According to the reconstruction requirement of the model, the relevant parameters were set and "High" was selected to improve the quality of the model. The other parameters were default values, which made the model more intuitive and realistic, and had higher simulation and visualization. Rotation, translation, enlargement, and reduction of the model were performed in the interface to understand the knee joint condition.

In the acute treatment of genu valgus, 3D printed osteotomy guide plate-assisted TKA does not require the conventional application of intramedullary and intramedullary positioning system, measurement of prosthesis size, and rotation of femur to measure the module. It is a relatively simple surgical operation and has a relatively short surgical time [23, 24]. The potential clinical advantage of time reduction includes the association of lower infection rates [25]. Since the medullary cavity opening is not required during the operation, the intraoperative and postoperative blood loss and the risk of fat embolism are reduced [26].

\section{Conclusion}

The present study showed that the operative time, intraoperative blood loss, and postoperative MFTA of patients in the combined treatment group were significantly lower than those in the treatment group, while the clinical and functional scores were significantly higher than those in the treatment group. These results indicate that the clinical effect of 3D printed osteotomy guide plate-assisted TKA in the treatment of valgus knee deformity is better than that of TKA alone, which is worthy of promotion. 


\section{Abbreviations}

2D: Two-dimensional; 3D: Three-dimensional; CAD: Computer-aided design; DICOM: Digital Imaging and Communications in Medicine; KSS: Knee Society Score; MFTA: Mean femorotibial angle; Mimics: Materialise's Interactive Medical Image Control System; RP: Rapid prototyping; TKA: Total knee arthroplasty; VCT: Volumetric computed tomography

\section{Acknowledgements}

Not applicable.

\section{Authors' contributions}

ZS carried out the study concepts, study design, manuscript editing, and manuscript review. HW was involved in the clinical studies. YD was dedicated to the literature research. JW carried out the definition of intellectual content and manuscript review. FW was involved in the data analysis and data acquisition. All authors have read and approved this article.

\section{Funding}

Guiyang Science and Technology Plan Project [2018] 1-80.

\section{Availability of data and materials}

The datasets used and analyzed during the current study are available from the corresponding author on reasonable request.

\section{Ethics approval and consent to participate}

This study was approved by the Ethics Committee of the authors' hospital, and informed consent was taken prior to enrollment (no. 2019081K).

\section{Consent for publication}

Not applicable.

\section{Competing interests}

The authors declare that they have no competing interests.

Received: 28 June 2019 Accepted: 28 August 2019

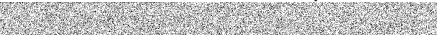

\section{References}

1. Bistolfi A, Massazza G, Rosso F, Deledda D, Gaito V, Lagalla F, et al. Cemented fixed-bearing PFC total knee arthroplasty: survival and failure analysis at 12-17 years. J Orthop Traumatol. 2011;12(3):131-6.

2. Nikolopoulos D, Michos I, Safos G, Safos P. Current surgical strategies for total arthroplasty in valgus knee. World J Orthop. 2015;6(6):469-82.

3. Helmy N, Dao Trong ML, Kühnel SP. Accuracy of patient specific cutting blocks in total knee arthroplasty. Biomed Res Int. 2014;2014:Article ID 562919. https://doi.org/10.1155/2014/562919.

4. Rossi R, Rosso F, Cottino U, Dettoni F, Bonasia DE, Bruzzone M. Total knee arthroplasty in the valqus knee. Int Orthop. 2014;38:273-83.

5. Hoekstra H, Rosseels W, Sermon A, Nijs S. Corrective limb osteotomy using patient specific 3D-printed guides: a technical note. Injury. 2016 47(10):2375-80

6. Victor J, Premanathan A. Virtual 3D planning and patient specific surgical guides for osteotomies around the knee: a feasibility and proof-of-concept study. Bone Joint J. 2013;95-B(11 Suppl A):153-8.

7. Rengier F, Mehndiratte A, von Tengg-Kobligk H, Zechmann CM, Unterhinninghofen R, Kauczor HU, et al. 3D printing based on imaging data: review of medical applications. Int J Comput Assist Radiol Surg. 2010;5(4):335-41.

8. Wang F, Zhu J, Peng X, Su J. The application of 3D printed surgical guides in resection and reconstruction of malignant bone tumor. Oncol Lett. 2017; 14(4):4581-4.

9. Ma L, Zhou Y, Zhu Y, Lin Z, Wang Y, Zhang Y, Xia H, Mao C. 3Dprinted guiding templates for improved osteosarcoma resection. Sci Rep. 2016;6:23335.

10. Keblish PA. The lateral approach to the valgus knee. Surgical technique and analysis of 53 cases with over two-year follow-up evaluation. Clin Orthop Relat Res. 1991;271:52-62.

11. Desseaux A, Graf P, Dubrana F, Marino R, Clavé A. Radiographic outcomes in the coronal plane with iASSIST ${ }^{\mathrm{TM}}$ versus optical navigation for total knee arthroplasty: a preliminary case. Orthop Traumatol Surg Res. 2016;102(3):363-8.
12. Schiraldi M, Bonzanini G, Chirillo D, de Tullio V. Mechanical and kinematic alignment in total knee arthroplasty. Ann Transl Med. 2016:4(7):130.

13. Park A, Nam D, Friedman MV, Duncan ST, Hillen TJ, Barrack RL. Interobserver precision and physiologic variability of MRI land-marks used to determine rotational alignment in conventional and patient-specific TKA. J Arthroplasty. 2015;30(2):290-5.

14. Matsuda S, Kawahara S, Okazaki K, Tashiro Y, Iwamoto Y. Postoperative alignment and ROM affect patient satisfaction after TKA. Clin Orthop Relat Res. 2013;471(1):127-33.

15. Ma B, Kunz M, Gammon B, Ellis RE, Pichora DR. A laboratory comparison of computer navigation and individualized guides for distal radius osteotomy. Int J Comput Assist Radiol Surg. 2014;9(4):713-24.

16. Oiao F, Li D, Jin Z, Gao Y, Zhou T, He J, Cheng L. Application of 3D printed customized external fixator in fracture reduction. Injury. 2015;46(6):1150-5.

17. Bäthis H, Perlick L, Tingart M, Lüring C, Zurakowski D, Grifka J. Alignment in total knee arthroplasty. A comparison of computer-assisted surgery with the conventional technique. J Bone Joint Surg Br. 2004;86(5):682-7.

18. Renson L, Poilvache P, Van den Wyngaert H. Improved alignment and operating room efficiency with patient-specific instrumentation for TKA. Knee. 2014:21(6):1216-20.

19. Olszewski R. Three-dimensional rapid prototyping models in craniomaxillofacial surgery: systematic review and new clinical applications. P Belg Roy Acad Med. 2013;2:43-77.

20. Ding HW, Shen JJ, Tu Q, Wang YJ, Zhang DH, Wang H, et al. Application of computer aided technique in osteoarthrosis. J Clin Rehabilit Tissue Eng Res. 2011:15(17):3113-8.

21. Arnal-Burró J, Pérez-Mañanes R, Gallo-Del-Valle E, Igualada-Blazquez C, Cuervas-Mons M, Vaquero-Martín J. Three dimensional-printed patientspecific cutting guides for femoral varization osteotomy: do it yourself. Knee. 2017;24(6):1359-68.

22. Shen C, Tang ZH, Hu JZ, Zou GY, Xiao RC, Yan DX. Patient-specific instrumentation does not improve accuracy in total knee arthroplasty. Orthopedics. 2015:38(3):e178-88.

23. Lionberger DR, Crocker CL, Chen V. Patient specific instrumentation. J Arthroplasty. 2014:29(9):1699-704.

24. Conteduca F, lorio R, Mazza D, Caperna L, Bolle G, Argento G, et al. Evaluation of the accuracy of a patient-specific instrumentation by navigation. Knee Surg Sports Traumatol Arthrosc. 2013;21(10):2194-9.

25. Thanni LO, Aigoro NO. Surgical site infection complicating internal fixation of fractures: incidence and risk factors. J Natl Med Assoc. 2004;96(8):1070-2.

26. Issa K, Rifai A, MCGrath MS, Callaghan JJ, Wright C, Malkani AL, et al. Reliability of templating with patient-specific instrumentation in total knee arthroplasty. J Knee Surg. 2013;26(6):429-33.

\section{Publisher's Note}

Springer Nature remains neutral with regard to jurisdictional claims in published maps and institutional affiliations.

Ready to submit your research? Choose BMC and benefit from:

- fast, convenient online submission

- thorough peer review by experienced researchers in your field

- rapid publication on acceptance

- support for research data, including large and complex data types

- gold Open Access which fosters wider collaboration and increased citations

- maximum visibility for your research: over $100 \mathrm{M}$ website views per year

At BMC, research is always in progress.

Learn more biomedcentral.com/submissions 